\title{
Different Characteristics of Radio Modules in Wireless Body Sensor Network Systems
}

\author{
Woosik Lee ${ }^{1}$, Min $\mathrm{Choi}^{2}$, and Namgi Kim ${ }^{1, *}$ \\ ${ }^{1}$ Computer Science Department, University of Kyonggi \\ Suwon, Kyonggi, Korea \\ ${ }^{2}$ University of Chungbuk \\ Heungdeok-gu, Chungbuk, Korea \\ \{wslee, ngkim\}@kgu.ac.kr, mchoi@cbnu.ac.kr
}

\begin{abstract}
Wireless body sensor network systems (WB-SNSs) can use diverse radio modules. However, previous studies did not consider different characteristics of radio modules, which deeply impact the performance of WBSNSs. In this paper, we analyze the performance of WB-SNSs using the representative radio modules $\mathrm{CC} 2420$ and $\mathrm{CC} 1000$. In this environment, we collected log data from real sensor devices deployed on the human body. After log data collection, we first show that $\mathrm{CC} 2420$ and $\mathrm{CC} 1000$ have different radio characteristics from diverse views, such as the received signal strength indication (RSSI) average and deviation, transmission power levels, and body movement. Through the analysis, we also find that an efficient transmission power control (TPC) algorithm should consider these diverse factors due to different radio modules.
\end{abstract}

Keywords: Wireless Body Area Network, Body Sensor System, Radio Module, CC2420, CC1000.

\section{Introduction}

As IT technologies are developed in pervasive computing, our society is moving toward ubiquitous computing environments. Sensor nodes in ubiquitous computing environments can collect diverse environmental information, such as temperature, humanity, light levels, and so forth. These sensors go beyond the bounds of wireless sensor networks (WSNs), toward wireless body sensor network systems (WB-SNSs). WB-SNSs can consist of various radio modules, such as CC2420, CC1000, and CC1010 [1]. These radio modules have unique characteristics, such as frequency, output power range, and radio propagation. The different characteristics of radio modules deeply impact the performance of WB-SNSs. These characteristics cause various problems, such as TPC failure, packet drops, and excessive energy consumption. Therefore, we must carefully consider the characteristics of diverse radio modules. In this paper, we analyze CC1000 and CC2420 radio modules, which

\footnotetext{
* Corresponding author.
} 
are representative radio modules in WB-SNSs. For analysis, we first investigated radio properties. We then show diverse results with RSSI values depending on transmission power (TP) levels. Finally, we analyze packet delivery rates (PDRs) and RSSI deviations depending on TP levels in various environments, such as standing, walking, and running.

\section{Related Work}

Many previous studies focused on particular radio modules [2]-[4]. Natarajan [2] and Shah [3] explored link layer characteristics of CC2420 by collecting log data from many volunteers in different environments. Quwaider [4] proposed a dynamic TPC algorithm using CC1000 radio modules at $433 \mathrm{MHz}$. However, these studies concentrated on particular radio modules, so their experimental results cannot be applied to other radio modules. On the other hand, Hamalainen [5] investigated a number of experimental data using SkyCross SMT-3TO10M-A antennas on a PHY layer for hospital environment channel modeling. However, this study did not consider either the MAC layer or other popular modules. The IEEE 802.15.6 working group [6] also investigated diverse channel models for WB-SNSs. However, they only concentrated on channel modeling and did not analyze diverse radio modules. Our previous work also only considered link channel characteristics using a CC2420 device [7]. Moreover, its work experiments took place in a static environment and did not consider dynamic environments such as walking and running. Thus, our previous study lacks additional experiments based on diverse radio modules in both static and dynamic environments. Therefore, in this paper, we investigate radio module properties and conduct experiments in real WB-SNSs so as to understand the different characteristics of diverse radio modules.

\section{Radio Properties Comparison}

In this section, we analyze radio properties of representative radio modules for WBSNSs, CC2420 and CC1000. Table 1 shows properties of both CC2420 and CC1000. CC2420 and CC1000 have the same RF modules, Zigbee. However, In terms of

Table 1. Properties of CC2420 and CC1000

\begin{tabular}{|c|c|c|}
\hline & CC2420 & CC1000 \\
\hline Frequency & $2.4 \mathrm{GHz}$ & $300-1000 \mathrm{MHz}$ \\
\hline Transmit Bit Rate & $250 \mathrm{kbps}$ & $76.8 \mathrm{kbps}$ \\
\hline Power Control & Programmable & Programmable \\
\hline $\begin{array}{c}\text { Output Power } \\
\text { Range }\end{array}$ & -24 to $0 \mathrm{dBm}$ & -20 to $10 \mathrm{dBm}$ \\
\hline RF Module & Zigbee & Zigbee \\
\hline Supply Voltage & 2.1 to 3.6 V & 2.1 to 3.6 V \\
\hline
\end{tabular}


frequency and transmit bit rate, $\mathrm{CC} 2420$ is higher frequency and transmit bit rate than CC1000. It means that $\mathrm{CC} 2420$ has more line-of-sight propagation property which can not travel over the horizon or behind obstacles. They also have different output power range. In other words, CC2420 and CC1000 have a different range of TP levels. So, same TP level maybe incur different TPC results.

\section{Experimental Environment}

Fig. 1 shows the experimental environment, such as sensor placements, the experiment area, and body movements. On the human body, the transmitting node is deployed on the chest and sends packets to the receiving node per $100 \mathrm{~ms}$. The receiving node is deployed on the stomach and stores a set of $\log$ data. The log data consist of 100 samples that include RSSI values, a power level index, and sequence numbers. After logging data, the host computer collects the log data in the EEPROM of the sensor node. Such setups apply equally to both $\mathrm{CC} 1000$ and CC2420. The right side of Fig. 1 shows our experimental area, the total size of which is $360900 \mathrm{~cm}$. Arrows in the figure indicate a moving path and a triangle shape is the starting point. In this environment, we repeatedly experimented with $\mathrm{CC} 1000$ and $\mathrm{CC} 2420$ radio devices for collecting raw data with respect to standing, walking, and running. A walking stride was approximately $45 \mathrm{~cm}$, and that of running was approximately $90 \mathrm{~cm}$.

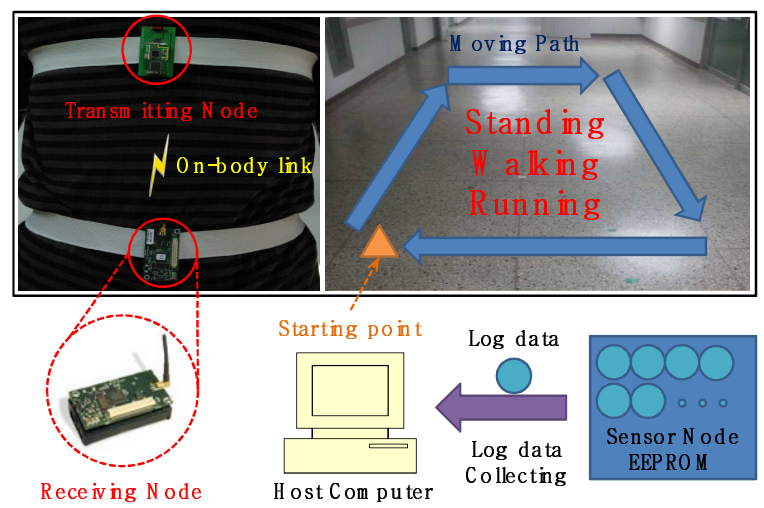

Fig. 1. Experimental environment

\section{$5 \quad$ Radio Transmission Comparison}

In Fig. 2, the x-axis represents TP levels and the y-axis RSSI values. As seen in the graph, the CC2420 TP levels are denser than those of CC1000. The range of CC2420 TP levels is from 3 to 31, but CC1000 is from 0 to 22. Through the graph, we see that CC2420 has an exponential shape and CC1000 a linear shape, depending on TP levels. Therefore, TPC algorithms using a linear equation for TP control cannot be applied to CC2420 radio modules. Second, CC1000 does not overlap each TP level, 
but CC2420 overlaps them from 4 to 8,8 to 12 , and 12 to 14 , as seen in Fig. 2. If CC2420 changes TP levels like CC1000, some TP levels will not work well. Thus, CC2420 should coarsely change TP levels for efficient energy management.

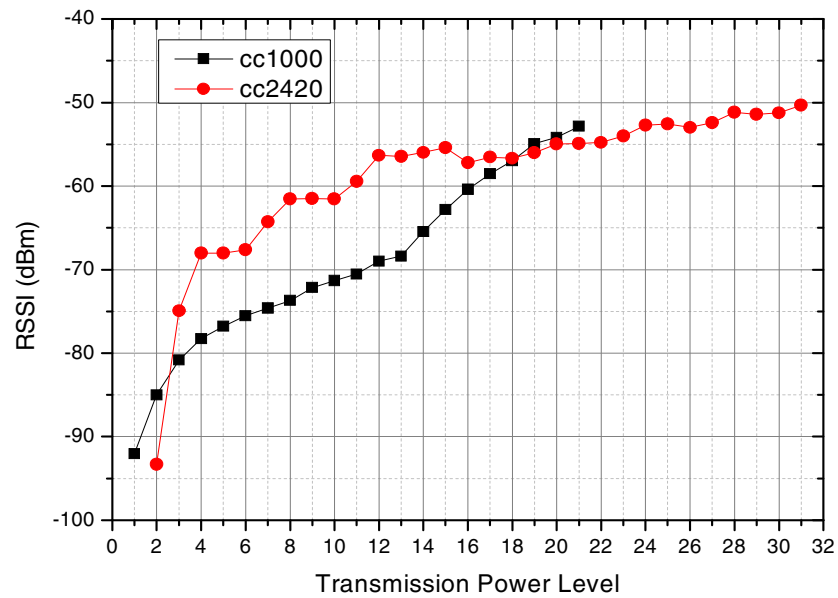

Fig. 2. Average RSSI of CC1000 and CC2420

\section{Packet Delivery Rate and RSSI Comparison}

Fig. 3 and 4 show total packet delivery rates as red bars and the CC2420 and CC1000 radio module RSSI values as black dots. In the graphs, the x-axis is the TP levels, the left $\mathrm{y}$-axis is the PDRs, and the right $\mathrm{y}$-axis is the RSSI values. These graphs consist of three situations: (a) standing, (b) walking, and (c) running. Through Fig. 3, we know that CC2420 has a lower PDR and higher RSSI deviations when a body is running. In this case, the RSSI deviation depending on TP levels is very large, making it difficult to estimate channel conditions. The fundamental reason for this is that standing is a static environment but running is a dynamic environment. In a static environment, the location of sensors does not change over time. On the other hand, in a dynamic environment, the sensor locations usually change as a result of the person's movements. Fig. 4 shows CC1000 experiment results, like Fig. 3 shows those of CC2420. However, CC1000 has a better PDR and fewer RSSI deviations than those of CC2420 in each situation. Moreover, CC1000 has a linear shape on the RSSI graph, while CC2420 has an exponential shape. Thus, CC1000 can apply to linear TPC algorithms, but CC2420 cannot apply. Furthermore, in dynamic environments, $\mathrm{CC} 1000$ has a higher PDR than CC2420 at low TP levels because CC2420 has more line-of-sight propagation than CC1000. Therefore, CC1000 can commute well, with low drop rates in dynamic environments. However, because $\mathrm{CC} 1000$ has a low transmit bit rate, we consider diverse radio modules for applications needing a high transmit bit rate. 


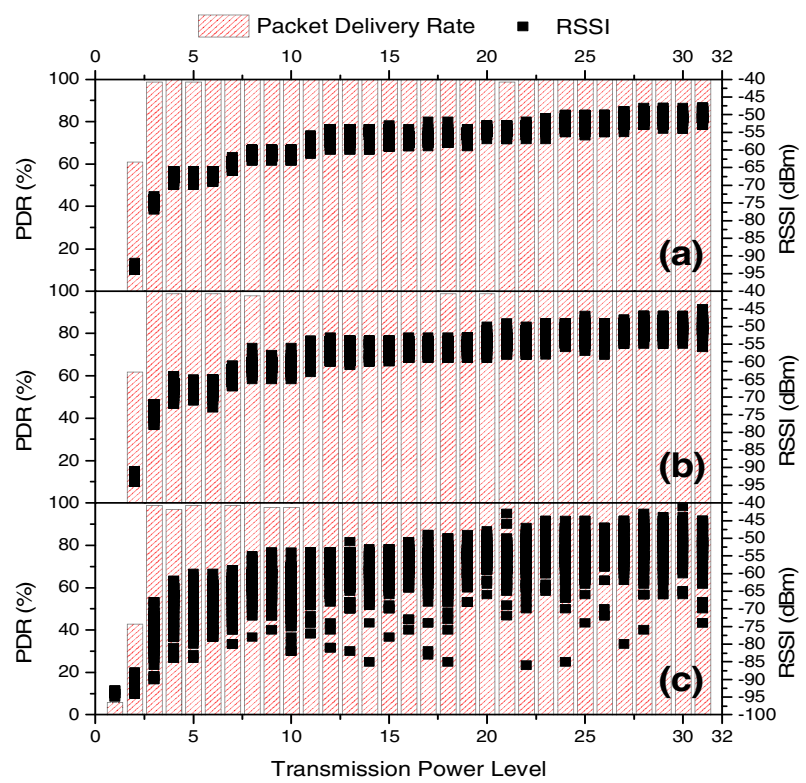

Fig. 3. CC2420 packet delivery rate and RSSI graphs when (a) standing, (b) walking, and (c) running

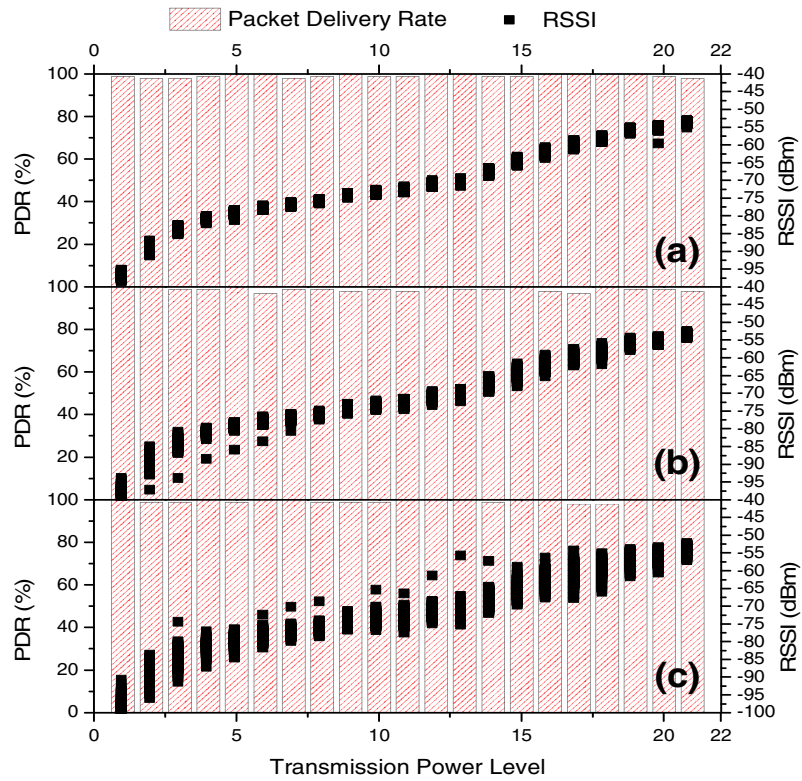

Fig. 4. CC1000 packet delivery rate and RSSI graphs when (a) standing, (b) walking, and (c) running 
Fig. 5 shows the RSSI deviations of CC1000 and CC2420. The X-axis shows the TP levels and the y-axis indicates RSSI values during three movements: standing, walking,

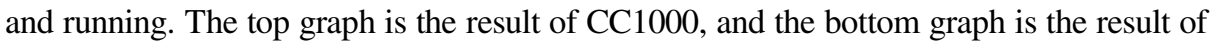
CC2420. In this graph, we compare the similarities and the differences between body movements in CC1000 and CC2420 modules. Both results show that almost all TP levels have similar results, in that walking has a higher deviation than standing, and running has a higher deviation than walking. This phenomenon is retained even if the TP levels are changed in both modules. Exceptionally, in the case of low TPs such as 1 and 2 in CC2420, the deviation is lower than other TP levels' deviations because of a lower PDR, as illustrated in Figure 3. For the other similarity, a different point depending on radio modules is that CC2420RSSI deviations are higher than those of CC1000 at all TP levels. Therefore, we must consider the differences between CC1000 and CC2420 modules when designing an efficient TPC algorithm.

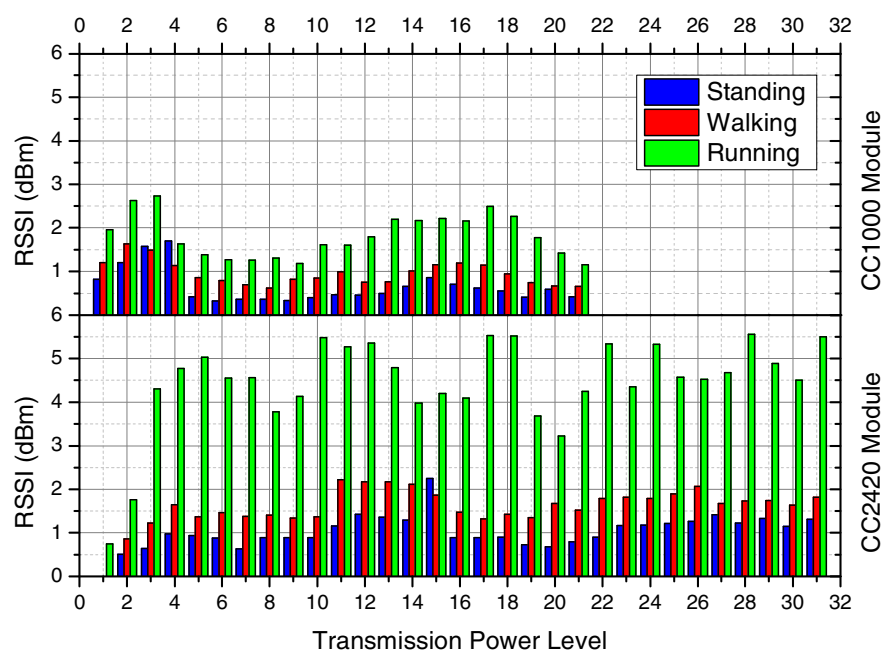

Fig. 5. RSSI deviation of CC1000 and CC2420

\section{Conclusion}

In this paper, we compare the characteristics of CC2420 and CC1000 radio modules through real experiments. To compare these modules, we first investigated radio properties such as the frequencies, transmit bit rates, power controls, output power ranges, RF modules, and supply voltages of CC2420 and CC1000. Second, we conducted experiments with real sensor nodes. Third, we analyzed the PDR and RSSI experimental results values for both the CC2420 and CC1000 modules. The results showed that CC1000 has a better PDR and less RSSI deviation than CC2420. Moreover, we knew that CC1000 has a linear RSSI shape, and that CC2420 has an exponential shape depending on TP levels. Lastly, the CC1000 and CC2420 RSSI deviations take similar forms according to the movements in this graph. However, the 
size of the deviations is different in that the CC2420 deviations are larger than those of CC1000. Through these experiments and the analysis, we knew that CC2420 and CC1000 modules have different properties, packet delivery rates, average RSSI values, and RSSI deviations. Consequently, we need a sophisticated TPC algorithm to cover the diverse characteristics of these radio modules. In the future, we will design a new TPC algorithm that can be simultaneously adopted for diverse radio modules.

Acknowledgment. This research was supported by Basic Science Research Program through the National Research Foundation of Korea (NRF) funded by the Ministry of Education, Science and Technology (grant number 2012R1A1A1002133)

\section{References}

1. Hall, P.S., Hao, Y.: Antennas and Propagation for Body-Centric Wireless Communications. Artech House (2006)

2. Natarajan, A., de Silva, B., Yap, K.K., Motani, M.: Link Layer Behavior of Body Area Networks at 2.4 GHz. In: MobiCom, pp. 241-252 (September 2009)

3. Shah, R.C., Nachman, L., Wan, C.Y.: On the Performance of Bluetooth and IEEE 802.15.4 radios in a body area network. BodyNets (March 2008)

4. Quwaider, M., Rao, J., Biswas, S.: Body-posture-based dynamic link power control in wearable sensor networks. IEEE Communications Magazine 48, 134-142 (2010)

5. Hamalainen, M., Taparugssanagorn, A., Iinatti, J.: On the WBAN radio channel modelling for medical applications. In: EUCAP, pp. 2967-2971 (April 2011)

6. Yazdandoost, K.Y., Sayrafian-Pour, K.: Channel Model for Body Area Network (BAN). doc: IEEE P802.15-08-0780-09-006 (April 2009)

7. Lee, W.S., Choi, M., Kim, N.: Experimental link channel characteristics in wireless body sensor systems. In: ICOIN, pp. 374-378 (February 2012) 